\title{
Improving Households Knowledge and Attitude on Water, Sanitation, and Hygiene Practices through School Health Programme in Nyakach, Kisumu County in Western Kenya
}

\author{
Job Wasonga, ${ }^{1}$ Charles Omondi Olang'o, ${ }^{2}$ and Felix Kioli ${ }^{2}$ \\ ${ }^{1}$ CARE International in Kenya, P.O. Box 88, Kisumu, Kenya \\ ${ }^{2}$ School of Arts and Social Sciences, Department of Sociology and Anthropology, Maseno University, Kenya \\ Correspondence should be addressed to Job Wasonga; jwasonga@hotmail.com
}

Received 25 July 2014; Revised 14 October 2014; Accepted 15 October 2014; Published 10 November 2014

Academic Editor: Kaushik Bose

Copyright (C) 2014 Job Wasonga et al. This is an open access article distributed under the Creative Commons Attribution License, which permits unrestricted use, distribution, and reproduction in any medium, provided the original work is properly cited.

The global problem of access to improved sanitation and water management practices has been compounded by the gap existing between knowledge and practice as well as attitude. The aim of this study was to assess households' knowledge and attitude on water, sanitation, and hygiene practices through a school health programme. Semistructured questionnaires, focus group discussions, key informant interviews, and observation checklist were used to obtain information from 95 households which were systematically sampled. It was found that a school programme may not improve the gap between knowledge, attitude, and practice but may be good for future generations. This was found to be due to sociocultural issues which impede hygiene transformation. The implication is that health programmes must find innovative ways of bridging this gap in order to bring change in households through culture sensitive interventions.

\section{Introduction}

The global problem of access to safe water and sanitation continues to plague the poor countries of the world. According to World Health Organization an estimated 2.6 billion people, comprising about 40 per cent of the world's population, live without adequate access to safe water and good sanitation [1]. About 2 billion of this population are found in rural areas of South Asia, Eastern Asia, and subSaharan Africa [1]. Majority of the affected population are found in informal settlements, periurban and rural parts of the developing countries where the practice of open defecation, poor sanitation services, and use of unsafe water persists because of knowledge gaps and attitude making people unable to practice basic hygiene. The existence of a gap between knowledge and practice on water, sanitation, and hygiene (WASH) despite intervention programmes being in places has also been associated with sociocultural factors which are seldom taken into account when intervention programmes are introduced [2]. Many of such interventions have also gone ahead to target schools and provide WASH services with the aim of influencing household adoption through children as proxies with mixed outcome.

The schools WASH intervention programmes in both developed and developing countries have been found to increase knowledge and improve behavioural practices in communities [3-5]. However, such practices are not sustainable, are inconsistent, and fizzle out after the intervention programme is over [6]. This has been found to be due to complex behavioural considerations influenced by social beliefs and culture which impedes attitude change despite availability of information or presence of knowledge [7]. An impact evaluation study conducted by BRAC WASH Programme in Bangladesh after a five-year intervention revealed that lack of awareness, knowledge, and hygiene practices were barriers to safe water use and improved sanitation due to a gap in knowledge and practice as a result of attitude and lack of motivation [2]. In this regard, we sought to find out the impact of a school WASH programme on knowledge diffusion and attitude change in the community of Nyakach in western Kenya. School WASH can be defined as promotion and provision of sanitation services such as adequate latrines 
in schools, promotion of safe water use, and imparting of lifelong skills that help in healthy practices among school children. The programme, Sustaining and Scaling School Water, Sanitation, and Hygiene plus Impact Community (SWASH+), was implemented in schools and communities with the aim of improving water, sanitation, and hygiene knowledge.

\section{The Study Area}

The study was conducted in Nyakach in Kisumu County. Nyakach is found in central Nyanza region which is predominantly inhabited by the Luo. It has 14 locations and 28 sublocations which form part of the Nyabondo plateau. There are 28 schools where SWASH+ was working, spreading across two of these sublocations. Nyakach is part of Nyando basin which is prone to displacement of population due to floods and other natural calamities like landslides. The area has unstable soil formation and little water sources. It also has low accessibility to water coverage and lack of adequate sanitation and water resource management [8]. Diarrhoea is a major cause of morbidity and mortality in this area contributing to deaths of children under 5 years of age which stands at 212/1000 births [8].

The area is inhabited by the Luo who are typically patrilineal and virilocal (living in man's family place). The Luo live in family homesteads, dala, which traditionally comprises a male head of the homestead, his wives or wife and their children, and his married son's families forming several household within the homestead [9]. The main economic activities of the people are subsistence agriculture and fishing as well as unorganized sand harvesting. The people also engage in migrant labour to contribute to family income [8].

Traditionally, like in any other parts of the Luoland, women do most of the farm work, helped by their children. Men clear the fields in preparation for planting of crops, build the houses and latrines, and earn money through fishing and trade although with modernization and high attrition rates women have also been engaged in fishing and trade. Girls look after their younger siblings, fetch water and firewood, help their mothers in the fields, cook, and clean while boys herd cattle, fetch firewood, and often catch fish. Many household tasks, however, are not gender-restricted, and boys and girls are often seen together working or caring for their younger siblings especially after leaving school [9]. The Luo consider their entire traditional way of life important and as a resource to be proud of. The social principles regarding age, kinship, and gender are very important in this community and therefore govern their existence and enculturation. The enculturation process involves the use of stories, legends, proverbs, riddles, and sometimes practical lessons such as fishing and ploughing using oxen. This process is majorly conducted by the grand mothers and fathers in the homestead although women played an important role in the education or enculturation process of children [10].

The Luo, are one of the major ethnic groups in Kenyan society and who for a very long time have been politically marginalized despite them being socially and politically conscious. Due to this marginalization, most of the health and development indicators are very low compared to other parts of the country. According to Kenya National Bureau of Statistics [11], around $48 \%$ of the population living in Kisumu county access water from an improved source with the majority getting there water from surface sources. The same report shows that $10 \%$ of the population lack sanitation with over 35\% using unimproved sanitation services. Although the situation has improved. This can be attributed to many nongovernmental organizations promoting safe water usage through point of use water quality improvement by use of bleach. The solution, Waterguard (1.5\% sodium hypochlorite), is popularly marketed by Population Services Kenya.

\section{Methods of Data Collection}

An observational and cross-sectional study targeting households was conducted among public primary schools where a water, sanitation, and hygiene intervention program was implemented. The intervention was implemented under Sustaining and Scaling School Water, Sanitation, and Hygiene plus Community Impact (SWASH+) program, an applied research by CARE International in Kenya, http://water.org/, Emory University and Great Lakes University of Kisumu [12]. The intervention was designed to identify, test, and bring to scale school water, sanitation, and hygiene (WASH) innovations as well as improve WASH situation in schools and communities in eight districts of Nyanza region in Kenya.

The study was conducted between May and June 2012, after six years of implementation and employed both quantitative and qualitative techniques. A systematic random sampling methodology was used to identify and interview 95 households situated around 4 public primary schools in Nyakach district. Semistructured questionnaires were used to gather information from the households through interviewing one member of the household found at that particular time.

An observation checklist was also used in these households to ascertain the WASH situation. This area (Nyakach district) was purposively selected from the other seven districts due to it not having participated in any SWASH+ conducted research and for logistical reasons. The previous research targeted schools that had less than recommended government (of Kenya) latrine to pupil ratio of 25:1 for girls and 30:1 for boys as well as schools that had water source within one kilometer during dry season. This research therefore, targeted the households within the implementing schools in this area which were never targeted in the previous larger study. This was also to avoid research fatigue as a result of conducting continuous studies among the population which the programme had done between the years 20062012. A detailed design of the previous study which informed this one is discussed elsewhere (see Freeman et al.) [12].

We collected data on sociodemographic characteristics, water sources, and methods of improving water quality at home, domestic water uses, sanitation, and hygiene practices as well as sources of knowledge on WASH. Observation checklist was also used for triangulation of the information 
TABLE 1: Sex of respondents, age distribution, and household membership.

\begin{tabular}{lcc}
\hline Characteristics & $\begin{array}{c}\text { Frequency } \\
(n=95)\end{array}$ & Percentage \\
\hline Sex & 12 & \\
Male & 83 & 12.6 \\
Female & & 84.4 \\
Level of education & 13 & \\
Illiterate & 14 & 13.8 \\
Lower primary & 41 & 14.9 \\
Upper primary & 11 & 43.6 \\
Lower secondary & 14 & 11.7 \\
Upper secondary & 1 & 14.9 \\
Tertiary/college & 1 & 1.1 \\
None response & & 0.0 \\
Age distribution & 8 & \\
$<20$ & 24 & 8.4 \\
$21-30$ & 21 & 25.3 \\
$31-40$ & 17 & 22.1 \\
$41-50$ & 12 & 17.9 \\
$51-60$ & 13 & 12.6 \\
$>61$ & & 13.7 \\
Household membership & 12 & 6.3 \\
$<3$ & 57 & \\
$4-6$ & 19 & \\
$7-10$ & & \\
$>11$ & & \\
& & \\
& &
\end{tabular}

given especially on water treatment where we tested drinking water for chlorine residual presence. We also observed the presence of latrine and dish rack, bathing, and handwashing facilities within the households. The data collection tools were pilot tested and revision was made where necessary before being used in the data collection for this study.

\section{Results}

4.1. Sociodemographic Characteristics of the Respondents. The majorities of the study respondents were female comprising $87.4 \%$ and were aged between 21 and 30 years. This was due to gender roles and men opting to let women be interviewed. The average household membership was between 4 and 6 people. The age distribution of the respondents based on gender and household membership, level of education, and literacy is shown in Table 1.

The respondents were asked about whether the programme has improved their knowledge and perception on water, sanitation, and hygiene practices; $50.7 \%$ said they had acquired no knowledge, $19.9 \%$ reported having acquired knowledge on water treatment, and $12.1 \%$ said they have acquired knowledge on water treatment and handwashing, $6.4 \%$ on handwashing hygiene alone, and $3.3 \%$ on latrine use or sanitation, while $3.3 \%$ were not sure (Table 2).
TABLE 2: Knowledge acquired as a result of the programme.

\begin{tabular}{lcc}
\hline Knowledge learned & Frequency & Percentage \\
\hline Water treatment & 19 & 19.9 \\
Latrine use & 3 & 3.3 \\
Handwashing (hygiene) & 6 & 6.4 \\
None acquired & 49 & 50.7 \\
Water treatment and handwashing & 11 & 12.1 \\
Other & 3 & 3.3 \\
None response & 4 & 4.2 \\
\hline
\end{tabular}

The study participants were also asked about their attitude measured using Likert type scale as a result of the intervention. The attitude was measured on a scale of highest to lowest among the respondents on water treatment, latrine use, handwashing, and water storage as a result of the WASH programme. The measurement was based on very high being most likely while very low being least likely (Table 3 ).

On water treatment $18 \%$ reported higher change of attitude, $9 \%$ reported high, $12.4 \%$ reported low, and $1.1 \%$ reported lower while $4.5 \%$ reported very low. On latrine use $19.3 \%$ reported very high, $11.4 \%$ reported higher, 9.1\% reported low, and $4.5 \%$ reported lower while $1.1 \%$ reported very low. On handwashing, $18.4 \%$ reported very high, $12.6 \%$ reported high, 9.2\% reported low, and $2.3 \%$ reported lower while $3.4 \%$ reported very low. On water storage, $18.4 \%$ reported very high, $11.5 \%$ reported high, and $5.7 \%$ reported low, while $8 \%$ reported lower.

Although the knowledge acquisition attributable to the programme was low as indicated in the above results, the change of attitude was evident from the responses captured. This was noted during focus group discussions as some of the respondents unanimously agreed.

There are changes-cases like cholera have reducedwe can say it is not there because of the education and mobilization of people to construct latrines and sanitation within the compound and handwashing after visiting latrine. All these changes came from the nongovernmental organizations and government officers. There are so many changes attributable to the programme, looking at how people lived before the program was introduced: we find that people now use latrines; there are dish racks and bathrooms. Then when we come to schools, you find that sanitation facilities and the level of health awareness has increased. This is because the children already know what to do and how to use the facilities. Then when we go back to the community, we find that we did not know a lot before. Like we did not know how to wash hands well. For now we have known that if we are washing our hands then you must wash well and even the wrist too. We need also to take time for handwashing which can also be a challenge as you can feel that it is a waste of too much timeso we can say that there are a lot of improvements. We can see these changes but it is not clear for us if they are there as a result of SWASH+ or not-but mostly the ones that children do, you find that they learn them in school, so they are things that they are taught in school and when they go back home they share with parents and parents also try to do them. 
TABLE 3: Attitude on water treatment and storage, latrine use, and handwashing.

\begin{tabular}{lcccc}
\hline Change of attitude & Water treatment $(n=95)$ & Water storage $(n=95)$ & Latrine use $(n=95)$ & Handwashing $(n=95)$ \\
\hline Higher & 16 & 49 & 30 & 18 \\
High & 8 & 16 & 15 & 13 \\
Low & 11 & 10 & 13 & 11 \\
Lower & 4 & 7 & 6 & 3 \\
Very low & 1 & 8 & 15 & 2 \\
None & 55 & 5 & & 48 \\
\hline
\end{tabular}

Although sometimes we say that when a nongovernmental organization comes to a community and does something, you would find that sometimes the name of the organization is not well known but what it has done is known by everyone. You might also find that only few people from that area know about it but what the organization is doing for them spreads within the whole community. So all these help in improving sanitation and reducing diseases.

The study examined the community source of water for domestic use, whose responsibility is to fetch water for household use, water usage at household, treatment, and storage methods.

4.2. Domestic Water Practices. According to the findings, women bear the brunt of fetching water comprising $56 \%$ while girls alone constituted $24 \%$ and mothers accompanied by their children constituted $20 \%$. Children fetching water alone constituted 2\%. Majority of the respondents, $75 \%$, fetch their water from the tap and $14 \%$ from the borehole while $11 \%$ from the river. In this community, water is mainly fetched in the morning as $62 \%$ of respondents alluded to, $31.2 \%$ said they fetch water at any time, and $4.2 \%$ said they fetch water in the evening while $1 \%$ fetch their water during daytime; $2.1 \%$ did not have preferred time of fetching water.

Although majority of the respondents, $92.7 \%$, have heard of safe water system and know about safe water storage containers (container with spigot and tap), it was observed that only $17.9 \%$ of households store their water in such containers. It was also observed that only $4.8 \%$ of the study participants had a storage container with a spigot with majority of them, $85.2 \%$, storing their water in containers with wide mouths. The use of a wide "mouth" traditional clay pot to store drinking water was common and was observed in almost all of the households in the study area. For them, the use of a pot makes the water cool and thus "sweet to drink."

Asked about their preferred method of household water treatment, $76.8 \%$ preferred Waterguard (1.5\% sodium hypochlorite solution), 8.4\% preferred P\&G Purifier of Water, a mixture of calcium hypochlorite and iron sulphate, $6.4 \%$ preferred other methods of water treatment, and $4.2 \%$ preferred Aquatabs (chlorine tablets) while $4.2 \%$ preferred boiling their drinking water. It was further observed that only $52.4 \%$ of the households had their drinking water containing residual chlorine when tested using N, N-diethylphenylenediamine (DPD) colorimetric method.

Water is used for many purposes in this community apart from drinking. Stored water is used for washing clothes and cooking, bathing, watering animals, religious purposes such as baptism, and curing illnesses. As was summed up by one of the participants during a key informant interview:

Water is Life. It has been blessed by God and it encompasses all that we do in this community. It makes us live our lives.

4.3. Sanitation and Hygiene Practices. When asked about the availability of latrine for fecal matter disposal, $80 \%$ of the respondents said they own one although the presence of latrine was observed in $78 \%$ of the households. The construction of latrine is majorly the work of the man of the household although in his absence, artisans are paid to construct one or the brothers' in-laws assist. It was observed that the most commonly used materials for latrine superstructure construction are old mosquito nets, reeds, polythene sheets, iron sheets, mud, bricks and cement mortar. Likewise, when asked about availability of bathroom, 74.7\% said they have one within the compound compared to $69.2 \%$ bathrooms which were observed. Those who do not have bathrooms take shower in the nearby river which also acts as a source of water for domestic use.

It was found that poor soil formation and economic reasons, apart from cultural practices, contribute to lack of these sanitary facilities in the households. In loose soils, construction of a latrine that can withstand the vagaries of weather requires additional resources since the construction starts from the pit. This involves lining of the pit from the base which is equivalent to building two latrines. The cost according to this community is too much and thus people build latrines which collapse during rainy season or resort to "cat method." This was noted by a respondent thus, "There are some people who are poor and where we are staying like now during the rainy season, latrines sink-they dig holes at night use them to defecate and cover with ash to reduce smell because-sometimes the holes are shallow." The study participants said that they are forced to build new latrines once the rainy season is over. This challenge can cause fatigue and make one not to have a latrine.

The study also revealed that handwashing with soap is marred by cultural beliefs and taboos which border around livestock rearing. Although availability of soap was observed in $77.9 \%$ of the households, only $7 \%$ had soap near the latrine for handwashing and use it in washing their hands after visiting the latrine despite $80 \%$ having knowledge on handwashing which they could demonstrate accurately and 
mentioned critical times to wash hands without any prompts. The source of information on handwashing was attributed to community health workers/clinics $41.5 \%$, media $23.4 \%$, and school children $20.2 \%$ while other sources were $9.6 \%$ and baraza or community gatherings $5.3 \%$.

The study also wanted to find out the method of household waste disposal. An observation of the presence of rubbish/garbage pit as well as dish rack was done. Across the entire community under study, only $37.1 \%$ rubbish pits were observed while only $59.6 \%$ dish racks were observed in the households visited.

\section{Discussion}

Measuring of human knowledge and attitude in response to specific interventions during implementation period of a programme may not give accurate information about behaviour change, but may be used to measure potential impact. This study gives a snap shot of the influence of an intervention programme on knowledge and attitude towards water use, sanitation, and hygiene practices among community members staying around intervention schools.

The findings of this study show that school WASH intervention may not necessarily make parents acquire knowledge on water treatment, latrine use, and handwashing with soap. These findings are supported by a previous study by [13] which found a statistical significance in message diffusion from schools to households as a result of the same programme. In this regard, we argue that there is more to learn from school WASH programme strategies of using children as health messages ambassadors to bridge the gap between knowledge and practice. Many factors as a result to sociocultural issues come into play in message delivery from children to their households targeting parents more than has been documented. The results show that there is need to understand the impact of enculturation on such factors as who fetches water and when, preference of certain water storage containers, and how they affect water use practices in specific cultural contexts. The use of mass media and community health volunteers could also be some of the probable means of message diffusion into the community.

As has been observed [14], people may be forced to comply with "having latrines" due to fear of social sanctions as a result of conformity. This study coincided with a campaign on community led total sanitation (CLTS) in the area which could have contributed to the high number of people with latrines made of polythene sheets, old mosquito nets, and reeds. CLTS is a social motivation strategy which ignites behavior change through facilitating communities by use of "shame and stigma" to construct latrines without reliance on any subsidy from outside [15]. Shame of not having a latrine or bathroom due to CLTS campaigns conducted by government officers to achieve open defecation free (ODF) status in the community as well as fear of social sanctions may have also contributed to people claiming to own latrines even if they do not, this could explain the difference in the reported number of households with latrines and bathrooms as compared to the number actually observed.
Sanitation goes beyond proper fecal matter disposal methods and includes wastes produced at the household and how they are disposed. This study therefore looked beyond latrine availability to the provision of garbage or refuse pits for waste disposal at the household level and availability of dish rack as a measure of good hygiene practice. Provision of these two facilities ensures that the health of the household members is preserved since they deny domesticated animals such as dogs from accessing wastes or utensils, thereby contaminating them. This may also help in preventing zoonotic and other vector borne related diseases. Lack of such facilities, therefore, may expose the inhabitants to diseases and encourages poor waste disposal methods or management as well as hygiene practices. A common method of domestic waste disposal in rural areas is by use of a garbage or rubbish pit, the presence of which can be used as a proxy indicator for positive health behavior.

Handwashing with soap as well as provision of water and soap next to the latrine encourages good hygiene behaviours as much as it is hard to sustain such behaviours within everyday settings [16]. This study reinforces these findings since soap was observed in $77.9 \%$ of the households yet only a paltry $7 \%$ provided the same next to the latrine and use soap to wash their hands. The study found that handwashing with soap is not common due to taboos and cultural practices which encourages those who rear livestock not to use soap for fear of losing their animals. Many studies have documented the benefits and challenges of soap provision and use $[6,17-$ 20] but few have examined the role of culture and belief system on handwashing behaviour. According to [21], norms, sociocultural representation, and hygiene practices always take place within social relations whose contributions are rarely documented. There is, therefore, need for an ethnographic study to identify some of these cultural practices which may act as a barrier to handwashing with soap as well as sanitation and water use. This will enable practitioners to come up with better strategies to combat disease spread and prevent diarrhea.

\section{Conclusion}

This study found that school WASH programme as much as it may help in knowledge diffusion to households, it does not necessarily lead to change of attitude especially on latrine use and handwashing with soap. In order to improve the gap that exists between knowledge and practice, there is need to carry out ethnographic studies to complement other studies and ensure that there is a deeper understanding of social settings that interventions take place. This will ensure better strategies for behavior change adoption interventions. Future research in school WASH should focus on ways of improving knowledge diffusion to household and bridge the gap of knowledge and practice. There is also need to come up with strategies of health education and promotion to ensure households understand the importance of waste disposal and management through use of rubbish pits and construction of dish racks to prevent diseases. 


\section{Ethical Approval}

The study was approved by the Maseno University Ethical Review Committee. The participants were asked for consent prior to gathering of information and anonymity as well as confidentiality was highly maintained while carrying out the study.

\section{Conflict of Interests}

The authors declare that there is no conflict of interests regarding the publication of this paper.

\section{Acknowledgments}

The authors wish to thank all the participants who took part in this study. The authors wish to sincerely thank Ben Okech, Eric Tindo, and Betty Ojeny of CARE Kenya, Rosebel Ouda, Sylvia Atieno, Dorothy Adhiambo, and Emily Awino who helped in data collection. The study was done under the umbrella of SWASH+ which was funded by Bill and Melinda Gates Foundation.

\section{References}

[1] World Health Organization, Progress on Sanitation and Drinking Water. 2010 Update, Geneva, Switzerland, 2010.

[2] Sifat-E-Rabbi and N. C. Dey, Impact of BRAC WASH-I Programme on Hygiene Knowledge and Practice in Rural Areas, Research Monograph Series no. 60, Research and Evaluation Division (RED), BRAC, Dhaka, Bangladesh, 2013.

[3] L. E. Greene, M. C. Freeman, D. Akoko, S. Saboori, C. Moe, and R. Rheingans, "Impact of a school-based hygiene promotion and sanitation intervention on pupil hand contamination in Western Kenya: a cluster randomized trial," American Journal of Tropical Medicine and Hygiene, vol. 87, no. 3, pp. 385-393, 2012.

[4] E. Blanton, S. Ombeki, G. O. Oluoch, A. Mwaki, K. Wannemuehler, and R. Quick, "Evaluation of the role of school children in the promotion of point-of-use water treatment and handwashing in schools and households-Nyanza Province, Western Kenya, 2007," The American Journal of Tropical Medicine and Hygiene, vol. 82, no. 4, pp. 664-671, 2010.

[5] C. E. O'Reilly, M. C. Freeman, M. Ravani et al., "The impact of a school-based safe water and hygiene programme on knowledge and practices of students and their parents: nyanza Province, western Kenya, 2006," Epidemiology and Infection, vol. 136, no. 1, pp. 80-91, 2008.

[6] S. M. Vindigni, P. L. Riley, and M. Jhung, "Systematic review: handwashing behaviour in low- to middle-income countries: outcome measures and behaviour maintenance," Tropical Medicine and International Health, vol. 16, no. 4, pp. 466-477, 2011.

[7] K. A. Mollah and T. Aramaki, "Social-epidemiological study for evaluation of water supply and sanitation systems of lowincome urban community in Dhaka, Bangladesh," Journal of Water and Health, vol. 8, no. 1, pp. 184-191, 2010.

[8] Government of Kenya, Nyando District Development Plan 2008-2012, Ministry of Planning, National Development and Vision 2030, Nairobi, Republic of Kenya, 2008.

[9] E. L. Grigorenko, P. W. Geissler, R. Prince et al., "The organisation of Luo conceptions of intelligence: a study of implicit theories in a Kenyan village," International Journal of Behavioral Development, vol. 25, no. 4, pp. 367-378, 2001.

[10] R. Prince and P. W. Geissler, "Becoming "one who treats": a case study of a Luo healer and her grandson in Western Kenya," Anthropology \& Education Quarterly, vol. 32, no. 4, pp. 447-471, 2001.

[11] Kenya National Bureau of Statistics, "Kisumu county multiple indicator cluster survey 2011," Final Report, Kenya National Bureau of Statistics, Nairobi, Kenya, 2013.

[12] M. C. Freeman, L. E. Greene, R. Dreibelbis et al., "Assessing the impact of a school-based water treatment, hygiene and sanitation programme on pupil absence in Nyanza Province, Kenya: a cluster-randomized trial," Tropical Medicine \& International Health, vol. 17, no. 3, pp. 380-391, 2012.

[13] S. Saboori, A. Mwaki, S. E. Porter, B. Okech, M. C. Freeman, and R. D. Rheingans, "Sustaining school hand washing and water treatment programmes: lessons learned and to be learned," Waterlines, vol. 30, no. 4, pp. 298-311, 2011.

[14] J. Bartram, K. Charles, B. Evans, L. O'hanlon, and S. Pedley, "Commentary on community-led total sanitation and human rights: should the right to community-wide health be won at the cost of individual rights?" Journal of Water and Health, vol. 10, no. 4, pp. 499-503, 2012.

[15] P. Harvey, "Community-led total sanitation, Zambia: stick, carrot or balloon?” Waterlines, vol. 30, no. 2, pp. 95-105, 2011.

[16] J. Davis, A. J. Pickering, K. Rogers, S. Mamuya, and A. B. Boehm, "The effects of informational interventions on household water management, hygiene behaviors stored drinking water quality and hand contamination in Peri-Urban Tanzania," The American Journal of Tropical Medicine and Hygiene, vol. 84, no. 2, pp. 184-191, 2011.

[17] A. Bowen, H. Ma, J. Ou et al., "A cluster-randomized controlled trial evaluating the effect of a handwashing-promotion program in Chinese primary schools," American Journal of Tropical Medicine and Hygiene, vol. 76, no. 6, pp. 1166-1173, 2007.

[18] B. Arnold, B. Arana, D. Mäusezahl, A. Hubbard, and J. M. Colford Jr., "Evaluation of a pre-existing, 3-year household water treatment and handwashing intervention in rural Guatemala," International Journal of Epidemiology, vol. 38, no. 6, pp. 16511661, 2009.

[19] W.-P. Schmidt, R. Aunger, Y. Coombes et al., "Determinants of handwashing practices in Kenya: the role of media exposure, poverty and infrastructure," Tropical Medicine and International Health, vol. 14, no. 12, pp. 1534-1541, 2009.

[20] S. Saboori, A. Mwaki, and R. D. Rheingans, "Is soapy water a viable solution for handwashing in schools?" Waterlines, vol. 29, no. 4, pp. 329-336, 2010.

[21] P. Hancart-Petitet, C. Dumas, A.-L. Faurand-Tournaire, A. Desclaux, and S. Vong, "Social and cultural dimensions of hygiene in Cambodian health care facilities," BMC Public Health, vol. 11, article 83, 2011. 

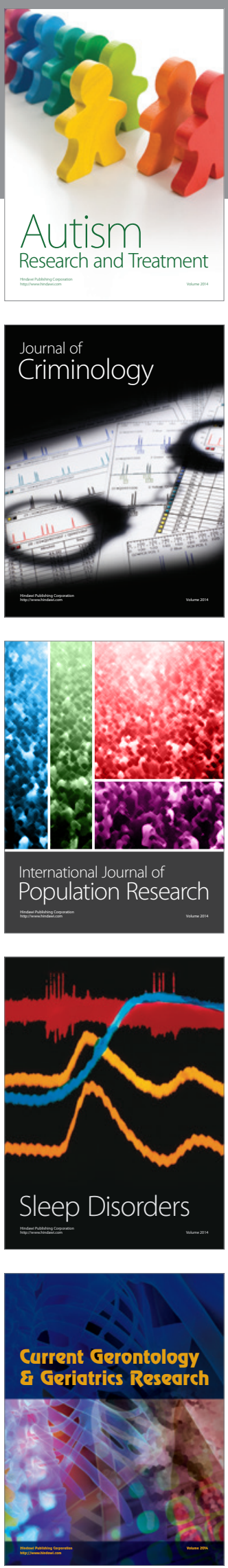
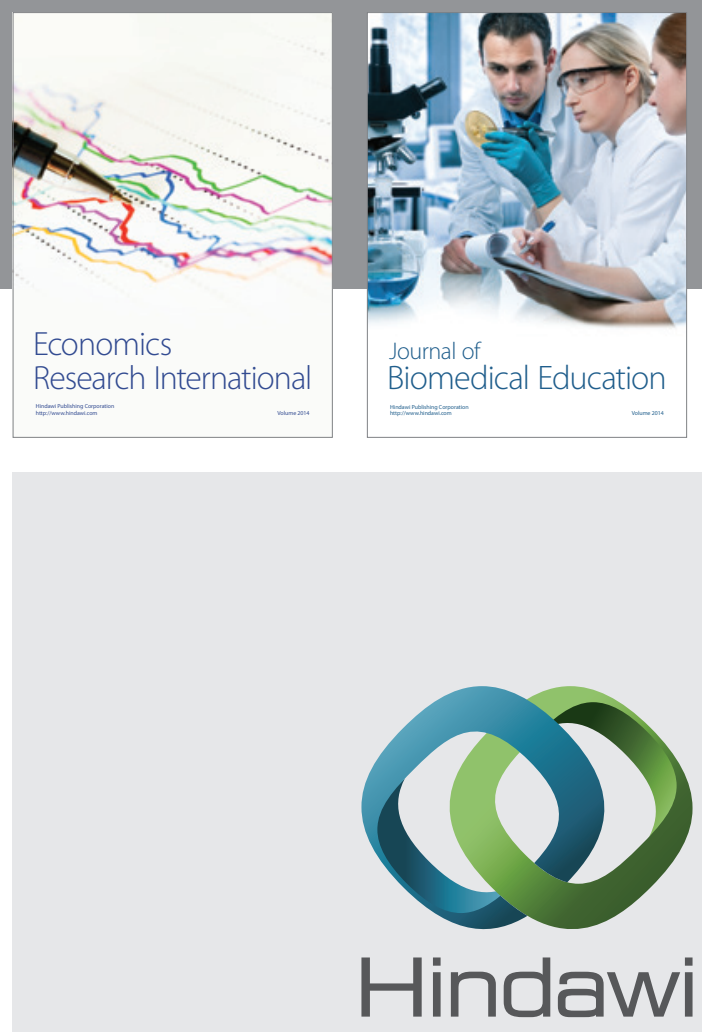

Submit your manuscripts at

http://www.hindawi.com
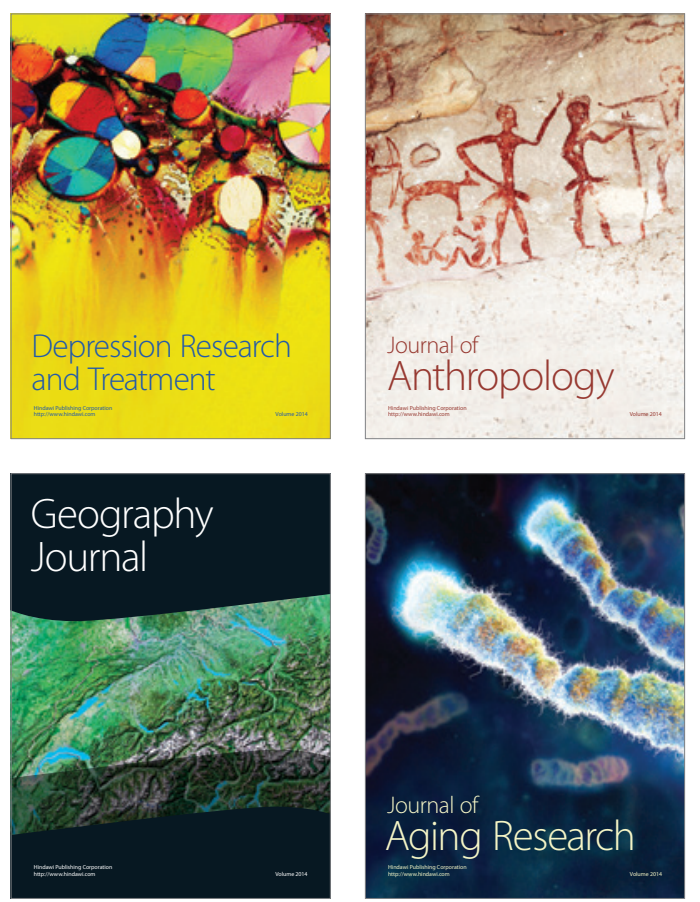
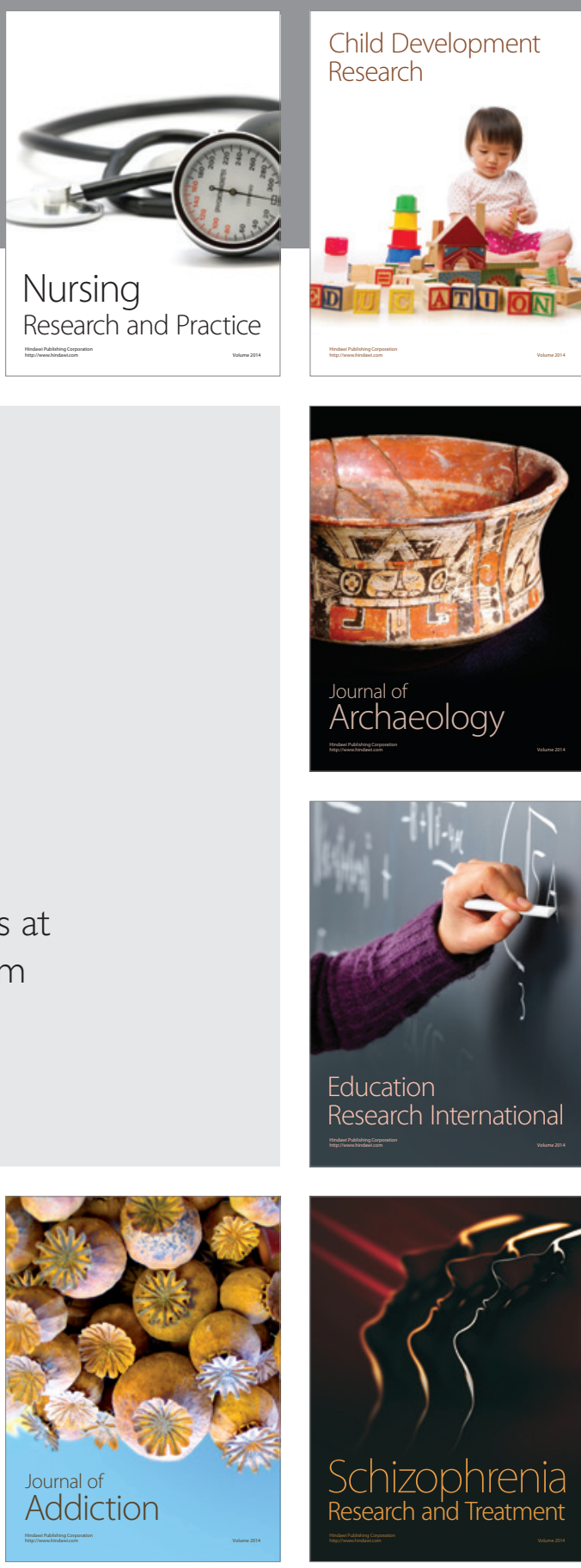

(D)
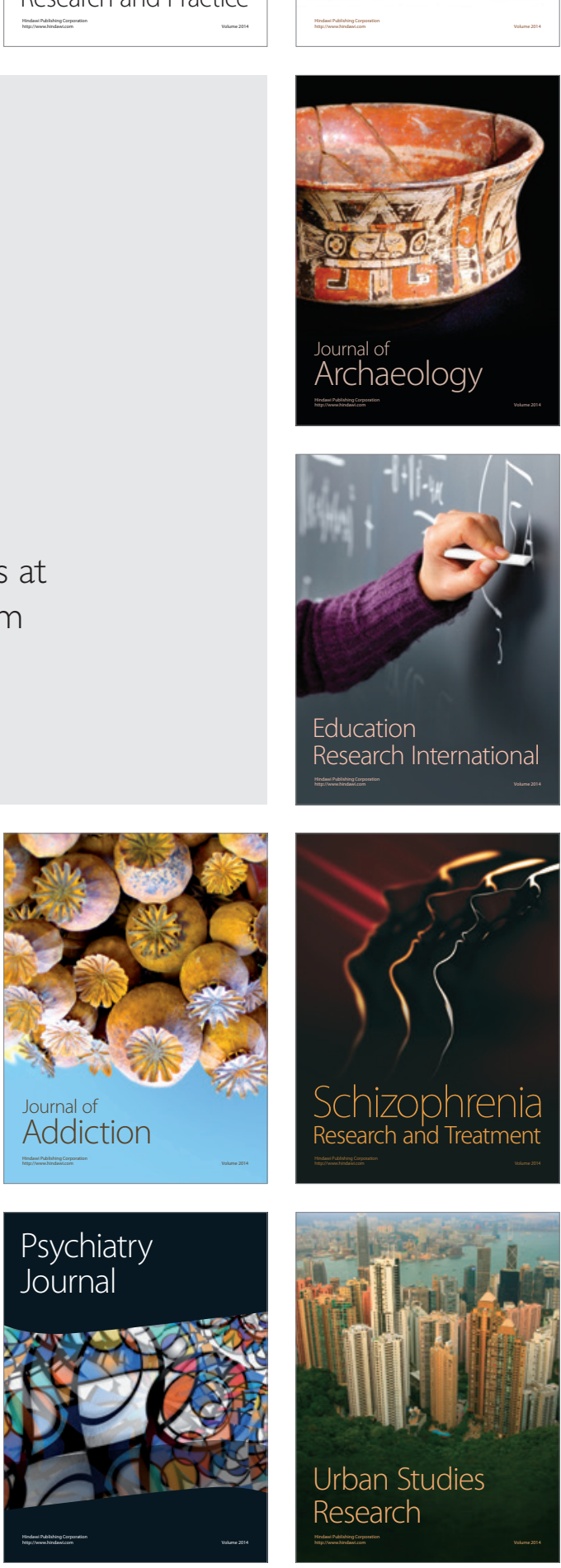